ORIGINAL ARTICLE

\title{
AST to platelets ratio (APRI) as a Biomarker of liver fibrosis status in patients of chronic Hepatitis B and Hepatitis C in comparison with Fibroscan
}

\author{
FARRUKH SALEEM ${ }^{1}$, SAIMA AMEER ${ }^{2}$, NIGHAT HAROON ${ }^{3}$, SAIRA BILAL ${ }^{4}$, WAJID ALI ${ }^{5}$, MAHM MUNIR AWAN $^{6}$ \\ ${ }^{1} M B B S$, Resident MD Radiology, Lahore General Hospital Lahore \\ ${ }^{2}$ FCPS Radiology, Professor and HOD of Radiology Department, Lahore General Hospital, Lahore \\ ${ }^{3}$ FCPS Radiology, Associate Professor of Radiology, Lahore General Hospital Lahore \\ ${ }^{4}$ FCPS Radiology, Associate Professor of Radiology, Lahore General Hospital Lahore \\ ${ }^{5}$ FCPS Radiology, Assistant Professor of Radiology, Lahore General Hospital Lahore \\ ${ }^{6}$ Associate Professor Radiology Nishtar Medical University Multan \\ Correspondence to: Dr. Farrukh Saleem, Email: drfarrinmc@gmail.com, Mobile No.: +92 3467505171
}

\begin{abstract}
Objective: The aim of the study is to correlate the AST to platelets ratio (APRI) with different stages of liver fibrosis measured by the fibroscan.

Methods: The study included 40 patients. The study was conducted in outpatient clinic of Lahore General Hospital. The study duration was from November-2020 to January-2021. Their fibroscan data was collected from hepatic clinic of general hospital OPD and their AST and Platelets values were obtained retrogradely from the PACS system of hospital. Correlation between the APRI and fibroscan values was done by linear Pearson correlation test.

Results: APRI values showed moderately positive correlation with increased kpa values and different stages of fibrosis $(r=0.55)$. Similarly, the correlation coefficient of AST also shown to be moderately positive correlated with kpa values and stages of fibrosis however this correlation was slightly weak as compared to APRI values ( $r=0.49)$. The correlation coefficient $(r)$ for platelets showed moderately negative correlation with liver fibrosis stages ( $r=-$ $0.43)$.

Conclusions: APRI levels increased as the degree of fibrosis and inflammation increased in patients suffering from chronic hep B and hep C. So, our data showed the clinical significance of APRI levels in diagnosing extent of liver fibrosis and cirrhosis.

Keywords: AST, Platelets, APRI, fibroscan, chronic hepatitis.
\end{abstract}

\section{INTRODUCTION}

Viral hepatitis, especially Hepatitis B \& Hepatitis C are very concerning health issue affecting the liver and resulting in widespread mortality and morbidity. The World Health Organization (WHO) estimates that in 2015, 257 million people worldwide had hepatitis B and 71 million people worldwide had hepatitis C. Both of these types of hepatitis can lead to chronic infection and hence decompensation and liver failures. It is also known that during the same year, 1.34 million people died from chronic and acute conditions affecting the liver caused by chronic viral hepatitis B \& C. ${ }^{1,2}$

Liver biopsy is usually considered as a gold standard in the measurement of fibrosis caused by many different chronic conditions. ${ }^{3}$ Chronic hepatitis $\mathrm{B}$ and $\mathrm{C}$ damages the hepatocytes and result in the elevation of serum levels of many enzymes. ${ }^{4}$ Many serum biomarkers of hepatic inflammation like ALT, AST, GGT and platelets levels have been in use to correlate the extent of fibrosis but AST to platelet ratio (APRI) shows the better correlation in determining the extent of fibrosis as compared to other biomarkers. ${ }^{5}$ Chronic inflammation of liver by viruses also result in decreased function of hepatocytes and eventually decreased levels of platelets. ${ }^{6}$ Platelets levels and function have also been used in evaluating the liver disease and as modulators of liver function. ${ }^{7}$

There are many noninvasive methods in evaluating the extent of fibrosis like transient elastography and shear wave elastography. ${ }^{8}, 9$ Fibroscan has the advantage over other noninvasive techniques as it is not dependent on operator so inter operator variability is very low and also very reproduceable, feasible \& cost effective. ${ }^{10}$ In this study we compared the APRI with kilopascal values generated by fibroscan in the measurement of fibrosis. In this study we correlated the APRI with liver fibrosis measured by the fibroscan.

\section{MATERIALS AND METHODS}

In this retrospective cross-sectional study, we included the data of 40 patients of hepatitis $C$ or hepatitis $B$ positive in whom fibroscan was done. The study was conducted in outpatient clinic of Lahore General Hospital. The study duration was from November-2020 to January-2021. Approval from hospital IRB was obtained. Any patient who had mass in the liver, or those having fatty texture or ascites were excluded.

Ultrasound was done on USG machine GE S8 to excluded any mass or other liver pathology. The data of AST and platelets count were collected from the PACS system of the hospital. AST to platelet ratio (APRI) is calculated from the following formula (AST/40 x100)/PLT.

All the data was analyzed using the SPSS 25.0. The correlation between the values of fibroscan measured in kilopascal(kpa), AST, PLT and APRI was done using the linear Pearson correlation test. 


\section{RESULTS}

The mean age of the patients was 39 ranging from 13 to 70 years. The study population was $30(75 \%)$ males and 10 $(25 \%)$ were females. There were 20 (8.0\%) patients infected with HBV and $32(80 \%)$ patients were infected with HBC. Mean values of AST, Platelets and APRI were 52, 226 and 0.75 respectively (Table 1 ).

Correlation of APRI, AST and Platelets levels with liver stages of fibrosis: Our data showed that mean levels of APRI, AST and Platelets increased with inflammation and fibrosis stages. The fibrosis measured by fibroscan is divided into four categories on basis of kpa values i.e., F0F1(kpa <7)-considered as No or minimal fibrosis, F2 \& F3 (kpa 7-11 for F2 and 11-14 for F3) as significant fibrosis and F4 (kpa >14) as cirrhosis. The mean values of APRI at above mentioned liver fibrosis stages was: FO-F1, F2, F3 and $\mathrm{F} 4$ were $0.35,0.75,0.8$ and 1.5 respectively.

Pearson correlation coefficient ( $r$ ) for APRI, AST and platelets were $0.55,0.49$ and -0.43 respectively with the $\mathrm{P}=<0.0001$. Hence APRI values showed moderately positive correlation with increased kpa values and different stages of fibrosis. Similarly, the correlation coefficient of AST also shown to be moderately positive correlated with kpa values and stages of fibrosis however this correlation was slightly weak as compared to APRI values. The correlation coefficient $(r)$ for platelets showed moderately negative correlation with liver fibrosis stages.

Table 1. Summary of data collected
\begin{tabular}{|l|l|l|}
\hline Age (years) & $39+/-11$ \\
\hline Gender & Male & $30(75 \%)$ \\
\cline { 2 - 3 } & Female & $10(25 \%)$ \\
\hline \multirow{3}{*}{ Fibrosis stage } & $0-1$ & $16(40 \%)$ \\
\cline { 2 - 3 } & 2 & $8(20 \%)$ \\
\cline { 2 - 3 } & 3 & $4(10 \%)$ \\
\cline { 2 - 3 } & 4 & $12(30 \%)$ \\
\hline AST & $52(24-138)$ \\
\hline Platelets & $226((50-495)$ \\
\hline APRI & $0.75(0.1-4.0)$ \\
\hline
\end{tabular}

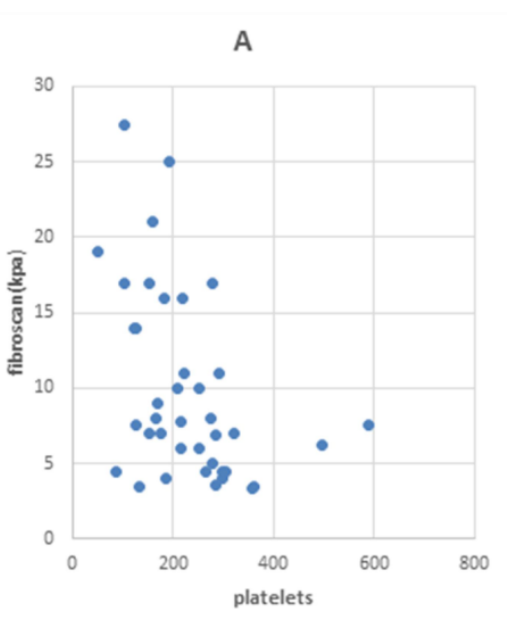

(A)

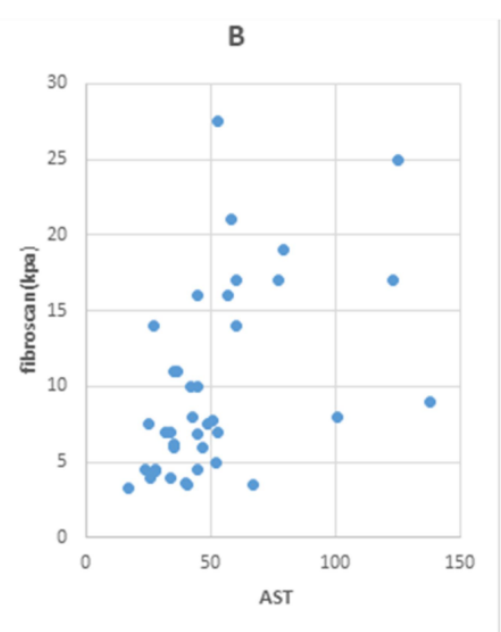

(B)

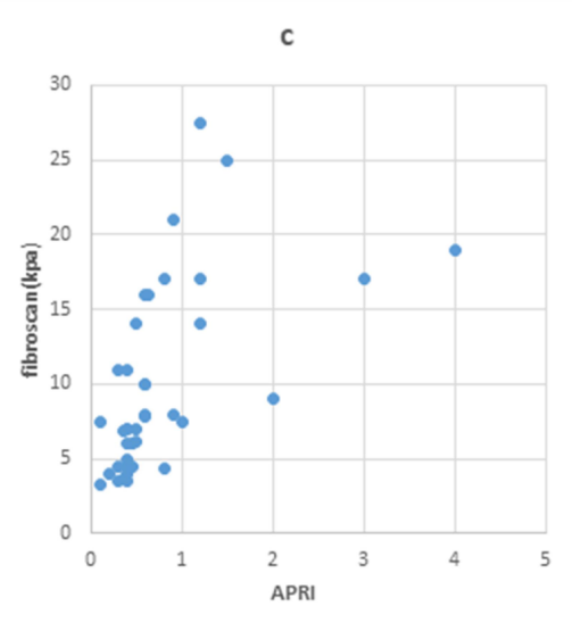

(C)

Figure 1. shows correlation between the Kpa values of fibroscan with different serum biomarkers. Fig. $1 \mathrm{~A}$ shows the correlation with platelets with $r$ (Pearson coefficient correlation) $=-0.43$. Fig 1B. shows correlation with serum AST levels with $r=0.49$. Fig 1C. shows correlation shows correlation with APRI with $r$ value of 0.55 . $P$ value for all above is 0.00 and $p<0.05$.

\section{DISCUSSION}

Result of this study shows that extent of liver damage had a clear effect on levels of APRI. There was significant correlation between APRI and stages of liver fibrosis. There was also slightly weaker but significant correlation between serum AST levels with stages of fibrosis. Platelets showed moderate negative correlation.

Age had little or no influence on APRI levels in this study. Similarly gender of the patients also showed no significant influence on APRI. However, the duration of infection had significant effect on APRI. As the duration of infection increases i.e., those patients who were infected with $\mathrm{HCV}$ or HBV earlier in life or for the longer period of life the damage to liver increased and also the APRI. Also, there was no significant difference in results in patients suffering from HBV or HCV hence the type of virus didn't significantly influence the APRI.
There has been focus on developing many novel biomarkers for hepatic fibrosis in hepatitis $C$ and hepatitis $B$ patients. ${ }^{11}$ Also, there are number of biomarkers like albumin, platelets, hyaluronic acid, ALT and AST have been evaluated however, no single biomarker which successfully predicts the fibrosis in HBV and HCV is not currently available. ${ }^{12}$ Also our data found that it's not the single entity rather than a combination of multiple serum biomarkers is required to successfully determine hepatic fibrosis.

Sripongpoun et al. conducted a study on accuracy of APRI score for predicting liver fibrosis. They reported that APRI value of $\geq 11$ has a sensitivity of $70.1 \%$, specificity $80.6 \%$ for diagnosis of liver cirrhosis. ${ }^{13}$

Jain et al. also reported a significant correlation between APRI values and Liver fibrosis. ${ }^{14}$ 
To our knowledge, it is the first study done in our region in which serum biomarkers like AST, Platelets and APRI are used against fibrosis of liver measured by fibroscan in patients of hepatitis B and C. Nevertheless, the study had some limitations. Firstly, it is done in one institution which limits the diversity of patients and secondly the sample size is relatively small. Finally, some patient's data used in this study may be suffering from the platelet's disease or taking anti platelets due to some other reason which is not taken into account as the data is collected retrogradely. Hence multiple other studies needed to be done at multiple hospitals with larger sample size to predict the prognostic value of APRI in liver fibrosis patients.

\section{CONCLUSION}

In this study we showed the significance of APRI by analyzing association between the AST, platelets levels and APRI with different stages of liver fibrosis in patients suffering from HBV and HCV levels. APRI increased as the stage of fibrosis increased. However, there is no single serum biomarker currently available which can predict the prognosis of liver fibrosis independently and hence the future aim will be to formulate a model comprising of multiple parameters to correctly assess the prognosis in liver fibrosis.

\section{REFERENCES}

1. World Health Organization. Global hepatitis report 2017: World Health Organization; 2017.

2. Kanda T, Goto T, Hirotsu Y, Moriyama M, Omata M. Molecular mechanisms driving progression of liver cirrhosis towards hepatocellular carcinoma in chronic hepatitis $b$ and c infections: a review. Int J Mol Sci. 2019;20(6).

3. Khalifa A, Rockey DC. The utility of liver biopsy in 2020. Curr Opin Gastroenterol. 2020;36(3):184-91.
4. Alavian SM, Showraki A. Hepatitis B and its Relationship With Oxidative Stress. Hepat Mon. 2016;16(9):e37973.

5. Lee S, Kim DY. Non-invasive diagnosis of hepatitis B virusrelated cirrhosis. World J Gastroenterol. 2014;20(2):445-59.

6. Kurokawa T, Ohkohchi N. Platelets in liver disease, cancer and regeneration. World J Gastroenterol. 2017;23(18):322839.

7. Lisman T, Luyendyk JP. Platelets as Modulators of Liver Diseases. Semin Thromb Hemost. 2018;44(2):114-25.

8. Xie LT, Yan CH, Zhao QY, He MN, Jiang TA. Quantitative and noninvasive assessment of chronic liver diseases using two-dimensional shear wave elastography. World $\mathrm{J}$ Gastroenterol. 2018;24(9):957-70.

9. Li Q, Huang C, Xu W, Hu Q, Chen L. Accuracy of FibroScan in analysis of liver fibrosis in patients with concomitant chronic Hepatitis B and nonalcoholic fatty liver disease. Medicine (Baltimore). 2020;99(23):e20616.

10. Iwaisako K, Brenner DA, Kisseleva T. What's new in liver fibrosis? The origin of myofibroblasts in liver fibrosis. J Gastroenterol Hepatol. 2012;27 Suppl 2(Suppl 2):65-8.

11. Lurie $Y$, Webb M, Cytter-Kuint R, Shteingart S, Lederkremer GZ. Non-invasive diagnosis of liver fibrosis and cirrhosis. World J Gastroenterol. 2015;21(41):11567-83.

12. Younesi S, Parsian H. Diagnostic accuracy of glycoproteins in the assessment of liver fibrosis: A comparison between laminin, fibronectin, and hyaluronic acid. Turk J Gastroenterol. 2019;30(6):524-31.

13. Sripongpun $\mathrm{P}$, Tangkijvanich $\mathrm{P}$, Chotiyaputta W, Charatcharoenwitthaya P, Chaiteerakij R, Treeprasertsuk S, et al. Evaluation of aspartate aminotransferase to platelet ratio index and fibrosis 4 scores for hepatic fibrosis assessment compared with transient elastography in chronic hepatitis C patients. JGH Open. 2020;4(1):69-74.

14. Jain $P$, Tripathi BK, Gupta B, Bhandari B, Jalan D. Evaluation of Aspartate Aminotransferase-to-Platelet Ratio Index as a Non-Invasive Marker for Liver Cirrhosis. Journal of clinical and diagnostic research : JCDR. 2015;9(11):Oc224. 